\title{
SPECTRAL DENSITY STUDY OF THE SU(3) DECONFINING PHASE TRANSITION ${ }^{\dagger}$
}

\author{
Nelson A. Alves ${ }^{1}$, Bernd A. Berg ${ }^{1,3} * *$ and Sergiu Sanielevici ${ }^{2,3}$ \\ ${ }^{1}$ Fakultät für Physik \\ Universität Bielefeld, D-4800 Bielefeld 1, Germany \\ ${ }^{2}$ Department of Physics \\ The Florida State University, Tallahassee, FL 32306, USA \\ ${ }^{3}$ Supercomputer Computations Research Institute \\ The Florida State University, Tallahassee, FL 32306, USA
}

\begin{abstract}
We present spectral density reweighting techniques adapted to the analysis of a time series of data with a continuous range of allowed values. In a first application we analyze action and Polyakov line data from a Monte Carlo simulation on $L_{t} L^{3}\left(L_{t}=2,4\right)$ lattices for the $\mathrm{SU}(3)$ deconfining phase transition. We calculate partition function zeros, as well as maxima of the specific heat and of the order parameter susceptibility. Details and warnings are given concerning i) autocorrelations in computer time and ii) a reliable extraction of partition function
\end{abstract}

$\dagger$ Submitted to Nuclear Physics B

** On leave of absence from Department of Physics, The Florida State University, Tallahassee. 
zeros. The finite size scaling analysis of these data leads to precise results for the critical couplings $\beta_{c}$, for the critical exponent $\nu$ and for the latent heat $\triangle s$. In both cases $\left(L_{t}=2\right.$ and 4$)$, the first order nature of the transition is substantiated. 


\section{INTRODUCTION}

Monte Carlo simulations of finite statistical systems at a coupling $\beta=\beta_{0}$ generate a time series of correlated data such that for appropriate observables $f$ the arithmetic average of measurements

$$
\bar{f}\left(\beta_{0}\right)=\frac{1}{N} \sum_{n}^{N} f_{n}
$$

becomes an estimator of the expectation value $<f>\left(\beta_{0}\right)$ :

$$
\hat{f}\left(\beta_{0}\right)=<f>\left(\beta_{0}\right)=\lim _{N \rightarrow \infty} \frac{1}{N} \sum_{n}^{N} f_{n} .
$$

Using the spectral density representation of the partition function

$$
Z(\beta)=\int_{S_{\min }}^{S_{\max }} d S n(S) \exp (\beta S),
$$

reweighting techniques allow to calculate the estimator $\bar{f}(\beta)$ for all $\beta$ in a sufficiently small neighborhood of $\beta_{0}$. Although reweighting techniques have a long history [1-9], the extent of practical improvements became only fully realized after the recent work by Ferrenberg and Swendsen $[7,8]$. In this paper we further elaborate on these techniques and cast them in a form suitable for practical applications in lattice gauge theories, where one has to deal with a time series of continuous variables. In particular, procedures for combining ("patching") data from simulations at various $\beta_{0}$ values are discussed in some detail. Altogether, we have in mind to establish a model analysis which may provide useful guidance for the analysis of data from future large scale simulations of the QCD deconfining phase transition, like the U.S. teraflop project [11]. Here, we give a reweighting analysis for the $\mathrm{SU}(3)$ deconfining phase transition. In a subsequent paper we shall present our $\mathrm{SU}(2)$ results. (From the point of view of reweighting techniques, the $\mathrm{SU}(2)$ theory turns out to be the more difficult case, because the action density distributions are barely distinguishable from Gaussian distributions.)

Pioneering numerical work on the $\mathrm{SU}(3)$ deconfining transition was done some years ago [12]. Renewed interest was stimulated by the APE collaboration [13], who raised doubts about the first order nature of the transition. This prompted a number of finite size scaling (FSS) studies [14-17] with the result that the first order nature of the transition was rather unambiguously established. Here, we give the details promised in [17]. 
Beyond [17] we present an investigation of autocorrelation times and a theoretical analysis concerning the numerical calculation of partition function zeros. The latter point enforces some corrections of previously stated results. Aside from quantities which are functions of the action (energy) [17], we also analyze now the Polyakov line susceptibility.

The paper is organized as follows: Section 2 introduces the spectral density method as used in this paper, section 3 investigates autocorrelations in computer time (for a review see [18]) and makes a connection to error bar calculations by binning [19,20], section 4 gives our reweighting calculations for the specific heat and a FSS estimate for the latent heat, section 5 is devoted to our calculation of partition function zeros and their FSS analysis. Beyond earlier approaches [5,21] a consistency argument is developed. In close analogy with work in [15] we give in section 6 a reweighting and FSS analysis for the Polyakov loop susceptibility. Summary and conclusions are contained in a final section 7 .

\section{SPECTRAL DENSITY MC CALCULATIONS}

We consider the SU(3) Wilson action

$$
S=\sum_{p} S_{p} \quad \text { with } \quad S_{p}=\frac{1}{3} \operatorname{Tr}\left(U_{p}\right) .
$$

where the sum goes over all plaquettes of the four-dimensional lattice with volume $V=$ $L_{t} L^{3}$. The MC simulation provides us with a time series of measurements for the action: $S_{n},(n=0, \ldots, N)$ where $N$ is the total number of measurements. We generate our data by means of the computer program published in ref. [22], taking measurements (meas.) after every sweep. A certain number of initial sweeps are omitted for thermalization (therm.). To have a convenient normalization, we monitor the corresponding action per plaquette (not to be confused with $S_{p}$ )

$$
s_{n}=\frac{S_{n}}{V_{p}}
$$

where $V_{p}=6 \mathrm{~V}$ is the total number of plaquettes. Let us first consider a single MC simulation at a fixed value $\beta=\beta_{0}$. What is the $\beta$ range of validity for a spectral density as obtained from the MC simulation at $\beta_{0}$ ? Let us assume it is valid for $\beta \in\left[\beta_{\min }, \beta_{\max }\right]$. In this range we are able to calculate estimators for quantities of physical interest by 


$$
\bar{f}(\beta)=\frac{F}{Z} \quad \text { with } \quad F=\sum_{n=1}^{N} f_{n} \exp \left(\triangle \beta S_{n}\right), Z=\sum_{n=1}^{N} \exp \left(\triangle \beta S_{n}\right) \text { and } \triangle \beta=\beta-\beta_{0} .
$$

For $\beta \neq \beta_{0}$ we call this reweighting. For technical reasons (floating point number overflows) one is actually forced to use in practice $S_{i}^{\prime}=S_{i}-\bar{S}\left(\beta_{0}\right)$ in these formulas. This contributes an irrelevant multiplicative factor and makes the arithmetic computationally feasible. With $f_{n}=\exp \left(i \beta_{y} S_{n}\right)$, the calculation of partition function zeros can be considered as a special case (where the numerator may be omitted in the one histogram case). For biased quantities the empirical error bar $\Delta \bar{f}$ of $\bar{f}$ is conveniently calculated by means of the jackknife method and one may also correct for the remaining bias (see [23] for details).

The relevant interval $\triangle \beta=\beta_{\max }-\beta_{\min }$ (reweighting range) will shrink with increasing volume like*

$$
\left.\triangle \beta \approx \sigma(s) \frac{d \beta}{d \hat{s}}\right|_{\beta=\beta_{0}}=\text { const } L^{-\rho / 2} L^{-d / 2}=\operatorname{const} L^{-1 / \nu},
$$

where $\rho=\alpha / \nu$ and the last equality made use of the hyperscaling relation [24] $\rho+d=2 / \nu$. We now have to determine the constant in (2.4). Essentially this boils down to the question: From which percentage of our total data at $\beta_{0}$ do we still expect meaningful results? Clearly, this depends somewhat on whether our statistics is large or small. $q$-tiles $s_{q}$ of our empirical action density with $q$ in the range 0.025 to 0.1 may still be expected to give meaningful answers. This has to be converted into a $\beta$-range. Let $q_{1}=q$ and $q_{2}=1-q$; we define $\beta_{\min }$ and $\beta_{\max }$ by:

$$
\beta_{\min }=\beta_{q_{1}} \quad \text { and } \quad \beta_{\max }=\beta_{q_{2}}
$$

where $\beta_{q}$ is given by the implicit equation

$$
\hat{s}\left(\beta_{q}\right)=s_{q} .
$$

This equation is solved numerically for $\beta_{q}$. Figures $1-3$ illustrate this procedure for the $4 \cdot 20^{3}$ lattice at $\beta_{0}=5.691$. Table 1 gives an overview of our SU(3) data including the $\beta_{\min }, \beta_{\max }$ and $s_{q_{1}}, s_{q_{2}}$ values for the choice $q=0.025$ (up to errors from conservative rounding of $\left.\beta_{\min }, \beta_{\max }\right)$. For $L_{t}=4$ Figure 4 depicts the $\triangle \beta(L)$ ranges versus $L$. Our

\footnotetext{
* Note that $\sigma(s)$, the standard deviation of $s$, goes as $L^{\rho / 2}$, while $\left.\frac{d \beta}{d \hat{s}}\right|_{\beta=\beta_{0}} \sim L^{-\rho}$.
} 
Table 1: Data and their reweighting ranges.

\begin{tabular}{|c|c|c|c|c|c|c|c|}
\hline$L_{t} \cdot L^{3}$ & therm. & meas. (indep.) & $\beta_{0}$ & $\beta_{\min }$ & $\beta_{\max }$ & $s_{q_{1}}$ & $s_{q_{2}}$ \\
\hline $2 \cdot 6^{3}$ & 10,000 & $120,000 \quad(360)$ & 5.094 & 5.026 & 5.1550 & 0.4060 & 0.4710 \\
\hline $2 \cdot 8^{3}$ & 10,000 & $120,000 \quad(120)$ & 5.090 & 5.042 & 5.1220 & 0.4080 & 0.4610 \\
\hline $2 \cdot 10^{3}$ & 10,000 & $120,000 \quad(30)$ & 5.090 & 5.050 & 5.1080 & 0.4090 & 0.4550 \\
\hline $2 \cdot 12^{3}$ & 10,000 & 120,000 & 5.092 & 5.064 & 5.1070 & 0.4110 & 0.4540 \\
\hline $2 \cdot 12^{3}$ & 10,000 & 120,000 & 5.095 & 5.078 & 5.1140 & 0.4130 & 0.4580 \\
\hline $4 \cdot 4^{3}$ & 10,000 & $120,000(1300)$ & 5.570 & 5.488 & 5.6710 & 0.4960 & 0.5560 \\
\hline $4 \cdot 4^{3}$ & 10,000 & $120,000(2080)$ & 5.610 & 5.533 & 5.7350 & 0.5120 & 0.5640 \\
\hline $4 \cdot 4^{3}$ & 10,000 & $120,000(3000)$ & 5.640 & 5.566 & 5.7650 & 0.5220 & 0.5690 \\
\hline $4 \cdot 6^{3}$ & 10,000 & $120,000(4000)$ & 5.500 & 5.449 & 5.5510 & 0.4830 & 0.5110 \\
\hline $4 \cdot 6^{3}$ & 10,000 & $120,000(1200)$ & 5.640 & 5.590 & 5.6880 & 0.5230 & 0.5520 \\
\hline $4 \cdot 6^{3}$ & 10,000 & $120,000(1400)$ & 5.645 & 5.598 & 5.6970 & 0.5240 & 0.5540 \\
\hline $4 \cdot 6^{3}$ & 10,000 & $120,000(1400)$ & 5.660 & 5.614 & 5.7170 & 0.5290 & 0.5570 \\
\hline $4 \cdot 6^{3}$ & 10,000 & $120,000(1600)$ & 5.690 & 5.641 & 5.7540 & 0.5380 & 0.5640 \\
\hline $4 \cdot 6^{3}$ & 10,000 & $120,000(2800)$ & 5.740 & 5.687 & 5.8090 & 0.5500 & 0.5720 \\
\hline $4 \cdot 8^{3}$ & 10,000 & $120,000(2900)$ & 5.600 & 5.567 & 5.6340 & 0.5160 & 0.5330 \\
\hline $4 \cdot 8^{3}$ & 10,000 & $120,000(800)$ & 5.670 & 5.638 & 5.7030 & 0.5350 & 0.5540 \\
\hline $4 \cdot 8^{3}$ & 10,000 & $120,000 \quad(700)$ & 5.693 & 5.661 & 5.7310 & 0.5420 & 0.5600 \\
\hline $4 \cdot 8^{3}$ & 10,000 & $120,000(1300)$ & 5.720 & 5.687 & 5.7630 & 0.5500 & 0.5650 \\
\hline $4 \cdot 10^{3}$ & 10,000 & $120,000(3000)$ & 5.600 & 5.575 & 5.6260 & 0.5180 & 0.5310 \\
\hline $4 \cdot 10^{3}$ & 10,000 & $120,000 \quad(550)$ & 5.680 & 5.656 & 5.7020 & 0.5400 & 0.5540 \\
\hline $4 \cdot 10^{3}$ & 10,000 & $120,000 \quad(350)$ & 5.693 & 5.671 & 5.7190 & 0.5440 & 0.5570 \\
\hline $4 \cdot 10^{3}$ & 10,000 & $120,000 \quad(600)$ & 5.710 & 5.687 & 5.7370 & 0.5490 & 0.5610 \\
\hline $4 \cdot 12^{3}$ & 10,000 & $120,000(3000)$ & 5.620 & 5.601 & 5.6380 & 0.5250 & 0.5340 \\
\hline $4 \cdot 12^{3}$ & 10,000 & $120,000 \quad(330)$ & 5.681 & 5.662 & 5.6960 & 0.5410 & 0.5510 \\
\hline $4 \cdot 12^{3}$ & 10,000 & $120,000 \quad(150)$ & 5.691 & 5.675 & 5.7080 & 0.5440 & 0.5550 \\
\hline $4 \cdot 12^{3}$ & 10,000 & $120,000 \quad(600)$ & 5.703 & 5.687 & 5.7230 & 0.5490 & 0.5580 \\
\hline $4 \cdot 14^{3}$ & 10,000 & $120,000 \quad(240)$ & 5.682 & 5.668 & 5.6930 & 0.5430 & 0.5510 \\
\hline $4 \cdot 14^{3}$ & 10,000 & $120,000 \quad(110)$ & 5.691 & 5.678 & 5.7030 & 0.5450 & 0.5540 \\
\hline $4 \cdot 14^{3}$ & 10,000 & $120,000 \quad(440)$ & 5.698 & 5.687 & 5.7130 & 0.5490 & 0.5560 \\
\hline $4 \cdot 16^{3}$ & 15,000 & $120,000 \quad(180)$ & 5.683 & 5.6711 & 5.6923 & 0.5428 & 0.5499 \\
\hline $4 \cdot 16^{3}$ & 15,000 & 120,000 & 5.691 & 5.6793 & 5.7006 & 0.5451 & 0.5536 \\
\hline $4 \cdot 16^{3}$ & 20,000 & $120,000 \quad(80)$ & 5.692 & 5.6814 & 5.7034 & 0.5455 & 0.5541 \\
\hline $4 \cdot 16^{3}$ & 15,000 & $120,000 \quad(200)$ & 5.697 & 5.6885 & 5.7096 & 0.5485 & 0.5557 \\
\hline $4 \cdot 20^{3}$ & 24,000 & $240,000 \quad(80)$ & 5.690 & 5.6820 & 5.6959 & 0.5454 & 0.5522 \\
\hline $4 \cdot 20^{3}$ & 22,000 & $240,000 \quad(65)$ & 5.691 & 5.6833 & 5.6975 & 0.5458 & 0.5528 \\
\hline $4 \cdot 20^{3}$ & 08,000 & 120,000 & 5.692 & 5.6842 & 5.6990 & 0.5460 & 0.5529 \\
\hline
\end{tabular}


subsequent analysis of autocorrelation times shows that for our present data the choice $q=0.025$ was too optimistic. However, this does not really matter, because the main purpose of calculating $\left[\beta_{\min }, \beta_{\max }\right]$ intervals first is to prevent reweighting calculations at absurd $\beta$ values.

In spin systems it is convenient to work with histograms. For lattice gauge theories the action varies typically over a continuous range and a histogram method is not recommendable for two reasons:

i) The size of the histogram bin (i.e. of the action interval deemed to constitute a single histogram entry) is an extraneous parameter. It is tedious to have to cope with it.

ii) Whatever the size of the bin, inevitably part of the information contained in the original sample gets lost. 
Instead of artificially introducing histograms, it is more convenient to rely directly on the empirical time series for the data. This requires to keep all measurements on disk or tape. In our present simulations we kept in double precision the spacelike and timelike plaquette expectation values and the real and imaginary Polyakov loop values. This amounts to up to $4 * 240,000 \mathrm{Real}^{*} 8$ data per $\left(L, \beta_{0}^{i}\right)$ simulation point, altogether filling up about 0.2 gigabyte of disk space (in unformatted storage). Consequently, the feasibility of this kind of analysis is tightly linked to the recent progress in storage technology and to the availability of large disks.

To cover a larger $\beta$ range one has to patch $\mathrm{MC}$ results from runs at different $\beta_{0}^{i}$ values $\left(\beta_{0}^{i+1}>\beta_{0}^{i}, i=1, \ldots, P\right)$, whose validity ranges overlap:

$$
s_{q 2}^{i+1}>s_{q_{2}}^{i}>s_{q_{1}}^{i+1}>s_{q 1}^{i} .
$$

Various methods can be found in the literature $[3,4,8,9]$. The two recent discussions $[8,9]$ both aim at minimizing the errors in the resultant estimates for $n(S)$. A crucial difference is that [9] fixes the needed relative normalizations of the histograms from data in the overlap regions only, whereas [8] exploits a self-consistency condition which was previously stated in [4]. The approach [8] yields results even if there is no overlap at all, whereas [9] cannot be applied in such a situation. For our purposes, results from patches without overlap are assumed to be meaningless and applying the self-consistency condition may be somewhat dangerous. For histograms with strong overlaps both methods will converge towards identical results.

More generally, it is useful to patch in such a way that the error of the actually calculated quantity is minimized. This leads to the following very straightforward approach, which we present in a form suitable for the time series analysis of our data. The first observation is that any combination

$$
\bar{f}(\beta)=\frac{\sum_{i=1}^{P} a_{i} F_{i}}{\sum_{i=1}^{P} a_{i} Z_{i}} \quad \text { with weight factors } a_{i}=a_{i}(\beta)>0
$$

is a valid estimator for $\langle f\rangle$. In the limit of infinite statistics each single histogram would yield the correct results. To simplify our considerations we impose the normalization

$$
\sum_{i=1}^{P} w_{i}=1 \quad \text { with } \quad w_{i}=a_{i} Z_{i}
$$

This converts equation (2.6) into 


$$
\bar{f}=\sum_{i=1}^{P} w_{i} \bar{f}_{i} \quad \text { with } \quad \bar{f}_{i}=\frac{F_{i}}{Z_{i}} .
$$

This equation makes clear that the optimal choice for the normalized weight factors $w_{i}$ is simply the inverse variance of $\bar{f}_{i}$

$$
w_{i} \sim \frac{1}{\sigma^{2}\left(\bar{f}_{i}\right)},
$$

and the overall constant is fixed by the normalization condition (2.7). In practical applications the exact variances $\sigma^{2}\left(\bar{f}_{i}\right)$ are unknown and we have to rely on the empirical error bars as estimators:

$$
w_{i} \sim \frac{1}{\left(\triangle \bar{f}_{i}\right)^{2}} .
$$

Of course, this just means that the standard way to add estimators weighted by their error bars is also the most suitable one for combining estimators from MC simulations at various $\beta_{0}$ values. However, several complications arise which deserve discussion.

i) Typically, our data exhibit large autocorrelation times (see next section). This limits a serious statistical analysis to using twenty jackknife bins. Imposing a $95 \%$ confidence limit (more precisely the $[0.025,0.975]$ confidence interval), the $\chi^{2}$ distribution [25] implies that $\left(\triangle \bar{f}_{i}\right)^{2} / \sigma^{2}\left(\bar{f}_{i}\right)$ fluctuates in the range $[0.58,2.13]$. Our experience is that the effect of these fluctuations on $\bar{f}(\beta)$ is harmless as long as only data sets from simulations at $\beta_{0}^{i}$ sufficiently close to $\beta$ are included. However, error bar fluctuations may become a serious problem if equation $(2.9 \mathrm{~b})$ is applied blindly. A highly recommendable cut-off is to set $w_{i}=0$ for $\beta \notin\left[\beta_{\min }, \beta_{\max }\right]$. It may be suitable to constrain the included data sets even further. For instance by excluding data sets which: a) taken alone give unsatisfactory results $\bar{f}_{i}(\beta)$ and $\mathrm{b}$ ) have $\beta$ located at one of the edges of the validity range.

ii) Once the weight factors are determined, error bars of $\bar{f}(\beta)$ from the combined statistics are not calculated by the standard rules of error propagation. Instead, new bins are formed, each relying on the combined statistics (2.6). If, after binning, autocorrelations still existed in the single data sets, they will become further reduced now as each new bin combines data from independent simulations. When appropriate, the new bins are used to construct jackknife bins in the standard way. 
iii) For connected estimators $\overline{f^{c}}=\bar{f}^{2}-\bar{f}^{2}$, like the specific heat or susceptibilities, it does not follow from equation (2.8) that the optimal weight factor is $w_{i} \sim 1 / \sigma^{2}\left(\bar{f}_{i}\right)$. The reason is that one has to calculate $\bar{f}^{2}$ according to $\bar{f}^{2}=\left(\sum_{i} w_{i} \bar{f}_{i}\right)^{2}$ and not according to $\bar{f}^{2}=\sum_{i} w_{i}\left(\bar{f}_{i}\right)^{2}$. But patching $\overline{f c}$ would require that $\overline{f^{2}}$ and $\bar{f}^{2}$ be calculated with identical weight factors. Fortunately however, this problem seems not to be too serious either. Weight factors calculated from $\bar{f}_{i}, \bar{f}^{2}{ }_{i}$ or $\bar{f}_{i}{ }_{i}$ should differ little. We have already noted that there are substantial statistical weight factor fluctuations which, in the reasonable range, do not influence the final result significantly. Therefore, we decided in favour of the simplest solution, namely to use the weight factors $w_{i} \sim$ $1 /\left(\triangle \bar{f}_{i}\right)^{2}$ for the calculation of $\overline{f^{c}}(\beta)$.

\section{AUTOCORRELATIONS IN COMPUTER TIME}

It has been emphasized in recent literature [18] that one has to control the integrated autocorrelation time $\hat{\tau}_{\text {int }}$, to be sure about the final error bars. However, in a typical realistic situation a Monte Carlo simulation may be perfectly reasonable with respect to all calculated quantities, including the confidence intervals implied by their error bars. And yet, $\hat{\tau}_{\text {int }}$ may remain the only quantity of interest that cannot be calculated reliably in such a simulation. It seems that previous investigations did not pay attention to this scenario and, therefore, we find it worthwhile to present some details.

To recall the concepts we first consider results obtained by a Metropolis generation of the Gaussian distribution. We generate $131,072=2^{17}$ numbers; due to a finite Metropolis stepsize, successive numbers are correlated. The integrated autocorrelation time is defined by:

$$
2 \tau_{\text {int }}\left(n_{b}\right)=\rho(0)+2 \sum_{i=1}^{n_{b}} \rho(i) \quad \text { with } \quad \rho(i)=\frac{\left\langle\left(s_{0}-\hat{s}\right)\left(s_{i}-\hat{s}\right)\right\rangle}{\left\langle\left(s_{0}-\hat{s}\right)\left(s_{0}-\hat{s}\right)\right\rangle}
$$

For $n_{b} \rightarrow \infty$ one recognizes that $2 \tau_{\text {int }}$ is just the ratio of the correct variance $\sigma^{2}(\bar{s})$ divided by the naive variance (obtained by using all events $s_{n}$ as if they were independent). A convenient way to calculate the correct variance is provided by binning [19]. The connection between binning and and the integrated autocorrelation time has been emphasized in [20]. Let us partition our data set into $N_{b}=2^{17-k}$ bins of length $n_{b}=2^{k}\left(N_{b}=32\right.$ for $\left.k=12\right)$ and denote the variance of $\bar{s}$ after the $k^{t h}$ binning by $\sigma_{k}^{2}(\bar{s})$. Note that 


$$
\sigma^{2}(\bar{s})=\lim _{k \rightarrow \infty} \sigma_{k}^{2}(\bar{s})
$$

whereas

$$
\sigma^{2}(s)=\sigma_{0}^{2}(s)
$$

Now

$$
\frac{\sigma_{k}^{2}(\bar{s})}{\sigma_{0}^{2}(\bar{s})}=\rho(0)+\frac{2\left(n_{b}-1\right)}{n_{b}} \rho(1)+\frac{2\left(n_{b}-2\right)}{n_{b}} \rho(2)+\ldots+\frac{2}{n_{b}} \rho\left(n_{b}-1\right),
$$

and for $n_{b} \rightarrow \infty$ the approach towards $2 \tau_{\text {int }}\left(n_{b}\right)$ follows from the rapid falloff of $\rho(i), i \rightarrow \infty$. We introduce the notation $\left(\triangle_{k} \bar{s}\right)^{2}$ for the estimators corresponding to $\sigma_{k}^{2}(\bar{s})$.

Using the Gaussian Metropolis data, figure 5 compares the increase of the variance under multiple binning, $\left(\triangle_{k} \bar{s}\right)^{2} /\left(\triangle_{0} \bar{s}\right)^{2}$, with the direct calculation of the integrated autocorrelation time. As expected, for sufficiently large $n_{b}$ identical values $(\approx 3.7)$ are approached. The convergence is somewhat better for the direct calculation of the integrated correlation time, whereas the binning procedure is computationally far more efficient. As usual in this paper, the error bars of $2 \tau_{\text {int }}$ are calculated with the double jackknife method [23]. However, for the error bar of the empirical variance $\left(\triangle_{k} \bar{s}\right)^{2}$ we do not use an estimator, but assume instead the $\chi^{2}$ distribution with $N_{b}-1$ degrees of freedom for $\left(N_{b}-1\right)\left(\triangle_{k} \bar{s}\right)^{2} / \sigma_{k}^{2}(\bar{s})$. The error bars depicted in the figures are then chosen such that twice their range, seen from the center, corresponds to a normal $95.4 \%$ confidence interval. The depicted symbols are the centers of these error bars and not the actually calculated estimators $\left(\triangle_{k} \bar{s}\right)^{2} /\left(\triangle_{0} \bar{s}\right)^{2}$ (due to the asymmetry of the $\chi^{2}$ distribution, these have somewhat lower values - which quickly approach the center as $\left.N_{b} \rightarrow \infty\right)$. If $n_{b}$ is large, the bins do not only become statistically independent, but the central limit theorem implies at the same time that they become normally distributed (each bin is an average over a large number of original data). For the normal (Gaussian) distribution it is well known [25] that the variance is $\chi^{2}$ distributed. Therefore, our assumption is justified whenever the statistical analysis is correct, i.e. sufficiently large $n_{b}$ values can be reached such that the correlations between the bins can indeed be neglected.

Figure 6 is the analog of figure 5, calculated with our SU(3) action data from the $4 \cdot 24^{3}$ lattice at $\beta_{0}=5.691$. Obviously, satisfactory convergence is not achieved. It is remarkable 
that direct calculation of the integrated autocorrelation time gives for large $n_{b}$ a much noisier estimator than we obtain from multiple binning. The enlargement of the first part of figure 6 in its upper left corner demonstrates this most clearly. Presumably binned data are favourably (de-)correlated. Consequently, we now rely on multiple binning. For even larger $n_{b}$ (small number of bins $N_{b}$ ) also the error bars of $\sigma_{k}^{2}(\bar{s}) / \sigma_{0}^{2}(\bar{s})$ increase rapidly. It is a perfectly possible scenario that $N_{b}=20$ bins are already sufficiently independent to allow a statistically reliable calculation of the action $\bar{s}$ (the $2 \sigma$ confidence level of the Student distribution with $N_{b}=20$ is almost normal), but a self-consistent calculation of the integrated autocorrelation time is impossible. The binning procedure sheds light on the reason. Let us assume we have $N_{b}=20$ independent bins. As already mentioned in this paper, it follows from the $\chi^{2}$ distribution with $N_{b}-1$ degrees of freedom that the $95 \%$ confidence interval of $(\triangle \bar{s})^{2} / \sigma^{2}(\bar{s})$ is $[0.58,2.13]$. In other words, the correct integrated autocorrelation time could be two times larger than the estimate or, equally well, only half the estimate. (Remember that the error bars given in the figures are half these confidence intervals.) To reduce this uncertainty to approximately $\pm 15 \%$, we need about $N_{b}=400$ independent bins. When, like in gauge theories or especially in full QCD, the computational effort is large, computer resources may only allow to create about $N_{b}=20$ independent bins. This may be fully sufficient to calculate quantities of physical interest with reliable confidence intervals. However, to compute the integrated autocorrelation time reliably requires a factor 20 more computer time. We conclude that the demand to calculate the integrated autocorrelation time in each investigation is exaggerated. Instead one may have to work under the assumption that the MC statistics is sufficiently large, for instance to give about $N_{b}=20$ independent bins, and then support this assumption with a number of consistency checks. For example, one may perform Student difference tests for estimators from independent MC runs (see the subsequent sections). Such an assumption of a sufficiently large statistics would be rather similar in spirit to other assumptions (for instance about the approach towards the continuum limit) already made for a typical lattice gauge theory simulation.

As one expects, the situation is under better control for our smaller systems. Figure 7 depicts the results from our $4 \cdot 6^{3}$ lattice at $\beta_{0}=5.640$. While a direct calculation of the integrated autocorrelation time remains inconclusive, its estimate from multiple binning is possible: $2 \hat{\tau}_{i n t}=100 \pm 10$ is consistent with all results from $k=9\left(N_{b}=234\right)$ on. Proceeding from smaller to larger lattices we obtain rough estimates of $2 \hat{\tau}_{\text {int }}$ for all our 
data sets. These results are included in table 1 by defining the number of independent (indep.) measurements as the number of measurements divided by $2 \hat{\tau}_{\text {int }}$. One has to caution that in the case of $L=20$ (240,000 original measurements) we can only rely on $N_{b}=14$ bins for estimating $2 \hat{\tau}_{i n t}$. This means an error of about $\pm 50 \%$ (around the center value) and no consistency checks towards higher $k$ values. The $L=24$ estimates may be unreliable altogether. However, for our subsequent purposes the achieved overall accuracy seems to be sufficient.

\section{SPECIFIC HEAT}

To characterize phase transitions, action (energy) fluctuations are particularly suitable. The variance of the action is related to the specific heat by $c_{V}=\beta^{-2} \sigma^{2}(S) / V$. The large $L$ finite size behaviour is

$$
\begin{gathered}
\sigma^{2}(S)=\left\langle\sum_{p}\left(S_{p}-\hat{S}_{p}\right) \sum_{p}\left(S_{p}-\hat{S}_{p}\right)\right\rangle \\
=\text { const } L^{\rho} \sum_{p}\left\langle\left(S_{p}-\hat{S}_{p}\right)^{2}\right\rangle=\text { const } L^{\rho} V_{p} \sigma^{2}\left(S_{p}\right) \quad \text { with } \rho=\frac{\alpha}{\nu}
\end{gathered}
$$

where $\sigma^{2}\left(S_{p}\right)$ is a constant bounded by $\left(S_{p}^{\min }-S_{p}^{\max }\right)^{2} / 4$. It is convenient to use the action density (2.1):

$$
\sigma^{2}(s)=V_{p}^{-2} \sigma^{2}(S)=\text { const } L^{\rho} V_{p}^{-1} \sigma^{2}\left(S_{p}\right)
$$

The exponent $\rho$ differentiates three interesting classes:

$$
\begin{cases}\text { 1. } & \rho=0 \text { for noncritical behaviour, } \\ \text { 2. } & 0<\rho<d \text { for second order critical behaviour, } \\ \text { 3. } & \rho=d \text { for a first order phase transition. }\end{cases}
$$

Here the given values of $\rho$ are defined as those obtained in the limit $L \rightarrow \infty$. It is convenient to introduce the notion of a strong second order phase transition for transitions with $\rho$ less but close to $d$ (for instance $\rho=2.99$ and $d=3$ ). For second and first order transitions, the subleading correction to equation (4.3) is assumed to be non-critical. This leads to the following FSS fits

$$
\sigma^{2}(s)=a_{1} L^{\rho-d}+a_{2} L^{-d} \text { for a second order phase transition }
$$


Table 2: Results from the action.

\begin{tabular}{|c|c|c|c|c|c|c|}
\hline$L_{t} \cdot L^{3}$ & $\beta_{0}$ & $\bar{s}$ & $\sigma_{0}^{2}(s)$ & $\sigma_{\max }^{2}(s)$ & bias & $\beta_{\max }$ \\
\hline $2 \cdot 6^{3}$ & 5.094 & $0.4390(10)$ & $3.4210^{-4}$ & $3.44(08) 10^{-4}$ & $11 \%$ & $5.0917(15)$ \\
\hline $2 \cdot 8^{3}$ & 5.090 & $0.4329(16)$ & $2.6710^{-4}$ & $2.70(07) 10^{-4}$ & $35 \%$ & $5.0908(10)$ \\
\hline $2 \cdot 10^{3}$ & 5.090 & $0.4249(26)$ & $2.0510^{-4}$ & 2.69 (11) $10^{-4}$ & $102 \%$ & $5.0928(11)$ \\
\hline $2 \cdot 12^{3}$ & 5.092 & $0.4282(31)$ & $2.3110^{-4}$ & $2.56(07) 10^{-4}$ & $155 \%$ & $5.0928(07)$ \\
\hline $2 \cdot 12^{3}$ & 5.095 & $0.4429(25)$ & $1.6110^{-4}$ & $2.62(31) 10^{-4}$ & $62 \%$ & $5.0928(10)$ \\
\hline $4 \cdot 4^{3}$ & 5.570 & $0.52781(48)$ & $2.5110^{-4}$ & $2.698(66) 10^{-4}$ & $4 \%$ & $5.5446(32)$ \\
\hline $4 \cdot 4^{3}$ & 5.610 & $0.54101(34)$ & $1.8410^{-4}$ & none & & \\
\hline $4 \cdot 4^{3}$ & 5.640 & $0.54822(23)$ & $2.2910^{-4}$ & none & & \\
\hline $4 \cdot 6^{3}$ & 5.500 & $0.49678(13)$ & $5.3210^{-5}$ & none & & \\
\hline $4 \cdot 6^{3}$ & 5.640 & $0.53732(25)$ & $5.9110^{-5}$ & $5.91(12) 10^{-5}$ & $6 \%$ & $5.638(09)$ \\
\hline $4 \cdot 6^{3}$ & 5.645 & $0.53905(20)$ & $5.9910^{-5}$ & $6.17(29) 10^{-5}$ & $3 \%$ & $5.614(14)$ \\
\hline $4 \cdot 6^{3}$ & 5.660 & $0.54322(18)$ & $5.6210^{-5}$ & none & & \\
\hline $4 \cdot 6^{3}$ & 5.690 & $0.55186(14)$ & $4.6610^{-5}$ & none & & \\
\hline $4 \cdot 6^{3}$ & 5.740 & $0.56188(12)$ & $3.4710^{-5}$ & none & & \\
\hline $4 \cdot 8^{3}$ & 5.600 & $0.52471(11)$ & $2.2510^{-5}$ & none & & \\
\hline $4 \cdot 8^{3}$ & 5.670 & $0.54429(21)$ & $2.5810^{-5}$ & 2.59 (07) $10^{-5}$ & $6 \%$ & $5.6713(36)$ \\
\hline $4 \cdot 8^{3}$ & 5.693 & $0.55098(23)$ & $2.3510^{-5}$ & $2.49(07) 10^{-5}$ & $10 \%$ & $5.6767(52)$ \\
\hline $4 \cdot 8^{3}$ & 5.720 & $0.55787(10)$ & $1.6510^{-5}$ & none & & \\
\hline $4 \cdot 10^{3}$ & 5.600 & $0.52458(07)$ & $1.1110^{-5}$ & none & & \\
\hline $4 \cdot 10^{3}$ & 5.680 & $0.54664(19)$ & $1.3610^{-5}$ & $1.406(50) 10^{-5}$ & $9 \%$ & $5.6876(25)$ \\
\hline $4 \cdot 10^{3}$ & 5.693 & $0.55119(17)$ & $1.2810^{-5}$ & $1.346(50) 10^{-5}$ & $6 \%$ & $5.6842(32)$ \\
\hline $4 \cdot 10^{3}$ & 5.710 & $0.55558(14)$ & $1.0210^{-5}$ & none & & \\
\hline $4 \cdot 12^{3}$ & 5.620 & $0.52998(05)$ & $6.3110^{-6}$ & none & & \\
\hline $4 \cdot 12^{3}$ & 5.681 & $0.54603(18)$ & $7.8510^{-6}$ & $9.23(55) 10^{-6}$ & $23 \%$ & $5.6909(30)$ \\
\hline $4 \cdot 12^{3}$ & 5.691 & $0.54990(27)$ & $9.3510^{-6}$ & $9.52(33) 10^{-6}$ & $21 \%$ & $5.6884(19)$ \\
\hline $4 \cdot 12^{3}$ & 5.703 & $0.55427(12)$ & $6.4210^{-6}$ & none & & \\
\hline $4 \cdot 14^{3}$ & 5.682 & $0.54637(19)$ & $5.4010^{-6}$ & $6.50(32) 10^{-6}$ & $28 \%$ & $5.6882(14)$ \\
\hline $4 \cdot 14^{3}$ & 5.691 & $0.54950(30)$ & $6.9910^{-6}$ & $7.14(36) 10^{-6}$ & $25 \%$ & $5.6924(13)$ \\
\hline $4 \cdot 14^{3}$ & 5.698 & $0.55281(13)$ & $4.5110^{-6}$ & none & & \\
\hline $4 \cdot 16^{3}$ & 5.683 & $0.54598(14)$ & $3.2110^{-6}$ & $4.95(42) 10^{-6}$ & $44 \%$ & $5.6902(15)$ \\
\hline $4 \cdot 16^{3}$ & 5.691 & $0.54923(28)$ & $5.1610^{-6}$ & $5.30(26) 10^{-6}$ & $28 \%$ & $5.6921(10)$ \\
\hline $4 \cdot 16^{3}$ & 5.692 & $0.54987(28)$ & $5.4010^{-6}$ & $5.47(26) 10^{-6}$ & $27 \%$ & $5.6916(10)$ \\
\hline $4 \cdot 16^{3}$ & 5.697 & $0.55245(13)$ & $3.4710^{-6}$ & none & & \\
\hline $4 \cdot 20^{3}$ & 5.690 & $0.54839(22)$ & $3.3210^{-6}$ & $3.90(16) 10^{-6}$ & $54 \%$ & $5.6918(6)$ \\
\hline $4 \cdot 20^{3}$ & 5.691 & $0.54912(28)$ & $3.8810^{-6}$ & 4.01 (15) $10^{-6}$ & $42 \%$ & $5.6915(6)$ \\
\hline $4 \cdot 20^{3}$ & 5.692 & $0.54918(32)$ & $3.6410^{-164}$ & $3.90(24) 10^{-6}$ & $43 \%$ & $5.6929(7)$ \\
\hline $4 \cdot 24^{3}$ & 5.691 & $0.54781(18)$ & $1.5610^{-6}$ & $4.4(1.7) 10^{-6}$ & $46 \%$ & $5.6934(9)$ \\
\hline $4 \cdot 24^{3}$ & 5.693 & $0.55101(18)$ & $1.9310^{-6}$ & $2.89(35) 10^{-6}$ & $46 \%$ & $5.6910(8)$ \\
\hline
\end{tabular}


and

$$
\sigma^{2}(s)=a_{1}+a_{2} L^{-d} \quad \text { for a first order phase transition. }
$$

The first order fit allows to determine the latent heat by the relation

$$
\triangle s=2 \sqrt{a_{1}}
$$

A weak first order transition is a first order transition with a small latent heat. Numerically it is difficult to distinguish a weak first order from a strong second order transition. The FSS scaling relations (4.4) are realized for $\beta=\beta_{c}$, where $\beta_{c}$ is the infinite volume critical coupling. In practice, the exact $\beta_{c}$ value is normally unknown. But we can construct the $L$ dependent series of $\beta_{\max }(L)$ values defined by

$$
\sigma^{2}(s ; L)(\beta)=\operatorname{maximum} \text { for } \beta=\beta_{\max }(L) \text {. }
$$

The corresponding $\sigma^{2}\left(s, \beta_{\max } ; L\right)$ is denoted by $\sigma_{\max }^{2}(s)$. Eqs. (4.4) are also satisfied for this series. In addition, this approach yields a precise estimate of $\beta_{c}$ through the fit

$$
\beta_{\max }(L)=\beta_{c}+\text { const } L^{-d}
$$

Exponentially small corrections to this fit are of relevance for small systems [7], but are better neglected [10] if the purpose is to estimate $\beta_{c}$. This is best done by fitting results from sufficiently large systems.

For notational simplicity we drop henceforth the distinction between estimators and exact theoretical definitions. Numerical results for each of our data sets are given in table 2. No stable values for $\sigma_{\max }$ are obtained when $\beta_{0}$ is too far from $\beta_{\max }$. Similarly, insufficient statistics may cause unstable behavior. The $4 \cdot 24^{3}$ lattice at $\beta_{0}=5.691$ is presumably an example. The discrepancies with [17] are only due to the fact that we now use 20 jackknife bins, whereas the error bars in [17] were estimated with respect to 32 jackknife bins (on the large systems a too generous number). The results for $\sigma_{\max }^{2}(s)$ are calculated by means of the double jackknife approach [23] and table 2 also lists the additional correction for the bias in $\%$ of the statistical error bar. Clearly, statistical error and bias of the entire statistics have a similar order of magnitude. For future reference we have also included the average action $\bar{s}=\bar{s}\left(\beta_{0}\right)$ and the variance $\sigma_{0}^{2}(s)$ at $\beta_{0}$ in table 2 . 
Table 3: Standard error propagation for the action results.

\begin{tabular}{c|c|c|c}
\hline$L_{t} \cdot L^{3}$ & $\sigma_{\max }^{2}(s)$, goodness & $\beta_{\max }$, goodness & $\#$ \\
\hline $2 \cdot 12^{3}$ & $2.56(07) 10^{-4}, 0.85$ & $5.0928(06), 0.94$ & 2 \\
& & & \\
$4 \cdot 4^{3}$ & $2.70(07) 10^{-4},-$ & $5.5446(32),-$ & 1 \\
$4 \cdot 6^{3}$ & $5.95(11) 10^{-5}, 0.41$ & $5.631(08), 0.16$ & 2 \\
$4 \cdot 8^{3}$ & $2.54(05) 10^{-5}, 0.32$ & $5.6730(30), 0.40$ & 2 \\
$4 \cdot 10^{3}$ & $1.38(04) 10^{-5}, 0.40$ & $5.6863(20), 0.41$ & 2 \\
$4 \cdot 12^{3}$ & $9.44(29) 10^{-6}, 0.65$ & $5.6891(16), 0.49$ & 2 \\
$4 \cdot 14^{3}$ & $6.78(24) 10^{-6}, 0.20$ & $5.6905(10), 0.03$ & 2 \\
$4 \cdot 16^{3}$ & $5.32(17) 10^{-6}, 0.57$ & $5.6916(07), 0.57$ & 3 \\
$4 \cdot 20^{3}$ & $3.95(10) 10^{-6}, 0.86$ & $5.6920(04), 0.29$ & 3 \\
$4 \cdot 24^{3}$ & $2.95(34) 10^{-6}, 0.40$ & $5.6921(06), 0.05$ & 2
\end{tabular}

Results are calculated from table 2 according to standard error progagation. The last column shows how many data sets were combined.

Using standard error propagation, we combine in table 3 for each lattice size the $\sigma_{\max }^{2}(s)$ and $\beta_{\max }$ estimates of table 2. Besides $\sigma_{\max }^{2}(s)$ and $\beta_{\max }$ the goodness of fit [26] is also listed. It is defined as the likelihood that the discrepancies between the estimates of table 2 (lattice size fixed) is due to chance. In case of two data sets we use the standard Student test [25] with $N=20$ data. For more than two data sets we rely on $\chi^{2}$ and assume a Gaussian distribution. If the assumptions are correct (of course there are slight corrections), the goodness of fit is a uniformly distributed random number between zero and one. Except for the goodness of fit for $\beta_{\max }$ from the $4 \cdot 14^{3}$ and $4 \cdot 24^{3}$ systems, all other values are reasonable. We tend to attribute the bad fit for the $4 \cdot 14^{3}$ to statistical fluctuations (after all we have a rather large number of systems), whereas a closer inspection (see below) of the $4 \cdot 24^{3}$ data sets gives rise to the suspicion that the assembled statistics is insufficient in this case.

Table 4 combines the action results by means of the patching method outlined in section 2. Within their statistical errors, the corresponding estimates of tables 3 and 4 are fully compatible. Only for the $4 \cdot 24^{3}$ lattices does patching reduce the error significantly. Figure 8 depicts the $\sigma^{2}(s, \beta)$ reweighting calculation for the single $4 \cdot 24^{3}$ lattices, whereas figure 9 shows the patched result. From these figures it is clear that the real improvement 
Table 4: Patching for the action results.

\begin{tabular}{l|c|l|l}
\hline$L_{t} \cdot L^{3}$ & $\sigma_{\max }^{2}(s)$ & \multicolumn{1}{|c}{$\beta_{\max }$} & \multicolumn{1}{|c}{$\#:$ weights. } \\
\hline $2 \cdot 12^{3}$ & $2.55(07) 10^{-4}$ & $5.0928(07)$ & $2: 0.67,0.33$. \\
& & & \\
$4 \cdot 4^{3}$ & $2.71(07) 10^{-4}$ & $5.5439(34)$ & $2: 0.76,0.24$. \\
$4 \cdot 6^{3}$ & $6.03(13) 10^{-5}$ & $5.624(11)$ & $2: 0.36,0.64$. \\
$4 \cdot 6^{3}$ & $6.03(16) 10^{-5}$ & $5.620(11)$ & $3: 0.30,0.53,0.17$. \\
$4 \cdot 8^{3}$ & $2.54(06) 10^{-5}$ & $5.6719(30)$ & $2: 0.61,0.39$. \\
$4 \cdot 10^{3}$ & $1.37(03) 10^{-5}$ & $5.6852(18)$ & $2: 0.45,0.55$. \\
$4 \cdot 12^{3}$ & $9.38(29) 10^{-6}$ & $5.6896(15)$ & $2: 0.34,0.66$. \\
$4 \cdot 14^{3}$ & $6.70(25) 10^{-6}$ & $5.6901(09)$ & $2: 0.48,0.52$. \\
$4 \cdot 14^{3}$ & $6.68(20) 10^{-6}$ & $5.6895(07)$ & $3: 0.39,0.44,0.17$. \\
$4 \cdot 16^{3}$ & $5.24(15) 10^{-6}$ & $5.6916(07)$ & $3: 0.17,0.41,0.43$. \\
$4 \cdot 16^{3}$ & $5.26(14) 10^{-6}$ & $5.6913(06)$ & $4: 0.15,0.35,0.36,0.14$. \\
$4 \cdot 20^{3}$ & $3.93(09) 10^{-6}$ & $5.6920(04)$ & $3: 0.38,0.35,0.27$. \\
$4 \cdot 24^{3}$ & $3.35(17) 10^{-6}$ & $5.6919(13)$ & $2: 0.42,0.58$.
\end{tabular}

The last column gives the number of patched data sets and the relative weights, ordered by increasing $\beta_{0}$. When the numbers match, the combined data sets are identical with those processed in table 3 . Otherwise, the validity ranges of table 1 make it clear which data sets have been added. All error bars are calculated with respect to twenty jackknife bins and corrected for the bias.

Table 5: $L_{t}=4$ FSS fits of $\sigma_{\max }^{2}(s)$

\begin{tabular}{c|ccc|ccc}
\hline & \multicolumn{3}{|c|}{ Standard error } & propagation $)$ & \multicolumn{3}{|c}{ (Patching) } \\
$L$ range & $10^{6} a_{1}$ & $10^{2} a_{2}$ & $Q$ & $10^{6} a_{1}$ & $10^{2} a_{2}$ & $Q$ \\
\hline $4-24$ & $2.22(09)$ & $1.26(02)$ & $10^{-23}$ & $2.22(08)$ & $1.27(2)$ & $10^{-25}$ \\
$6-24$ & $2.39(09)$ & $1.20(02)$ & 0.40 & $2.42(08)$ & $1.18(2)$ & 0.25 \\
$8-24$ & $2.47(10)$ & $1.17(03)$ & 0.76 & $2.49(09)$ & $1.15(3)$ & 0.81 \\
$10-24$ & $2.48(12)$ & $1.16(04)$ & 0.64 & $2.52(10)$ & $1.14(3)$ & 0.84 \\
$12-24$ & $2.39(14)$ & $1.22(06)$ & 0.77 & $2.46(12)$ & $1.17(5)$ & 0.91 \\
$14-24$ & $2.41(18)$ & $1.21(10)$ & 0.58 & $2.51(14)$ & $1.14(8)$ & 0.97
\end{tabular}

These fits use eq. (4.4b): thus, a first order transition is assumed.

due to patching is in fact much more impressive than noticeable from the $\sigma_{\max }^{2}(s)$ error bar reduction in table 4 as compared with table $3\left(4 \cdot 24^{3}\right.$ lattice). For our other data sets the 
Table 6: $L_{t}=4 \sigma^{2}(s)$ FSS fits of $\beta_{\max }(L)$

\begin{tabular}{c|ccc|ccc}
\hline & \multicolumn{2}{|c|}{ (Standard error propagation) } & \multicolumn{3}{|c}{ (Patching) } \\
$L$ range & $\beta_{c}$ & $a$ & $Q$ & $\beta_{c}$ & $a$ & $Q$ \\
\hline $4-24$ & $5.6934(3)$ & $-09.54(21.0)$ & 0.20 & $5.6933(3)$ & $-09.60(22.0)$ & 0.15 \\
$6-24$ & $5.6934(4)$ & $-10.00(01.0)$ & 0.15 & $5.6936(4)$ & $-10.60(01.1)$ & 0.13 \\
$8-24$ & $5.6931(4)$ & $-08.40(01.2)$ & 0.60 & $5.6933(4)$ & $-09.30(01.2)$ & 0.58 \\
$10-24$ & $5.6928(5)$ & $-06.10(01.8)$ & 0.98 & $5.6929(5)$ & $-07.60(01.7)$ & 0.80 \\
$12-24$ & $5.6927(5)$ & $-05.60(02.6)$ & 0.96 & $5.6929(6)$ & $-07.50(02.5)$ & 0.65 \\
$14-24$ & $5.6926(6)$ & $-04.90(03.4)$ & 0.89 & $5.6932(7)$ & $-09.30(03.1)$ & 0.66
\end{tabular}

The $\beta_{c}$ are extracted using eqs. (4.6) and (4.7).

Table 7: $L_{t}=2$ FSS fits of $\sigma_{\max }^{2}(s)$ and $\beta_{\max }(L)$

\begin{tabular}{c|ccc|ccc}
\hline & \multicolumn{2}{|c|}{ Standard error propagation) } & \multicolumn{3}{|c}{ (Patching) } \\
$L$ range & $10^{4} a_{1}$ & $10^{2} a_{2}$ & $Q$ & $\beta_{c}$ & $a$ & $Q$ \\
\hline $6-12$ & $2.39(07)$ & $2.17(25.0)$ & 0.14 & $5.0929(07)$ & $-0.44(38.0)$ & 0.36 \\
$8-12$ & $2.53(10)$ & $0.94(71.0)$ & 0.48 & $5.0937(09)$ & $-1.43(84.0)$ & 0.61 \\
$10-12$ & $2.38(23)$ & $3.10(03.1)$ & - & $5.0928(20)$ & $0.00(03.0)$ & -
\end{tabular}

Fits to $\sigma_{\max }^{2}$ use eq. (4.4b); the $\beta_{c}$ are extracted using eqs. (4.6) and (4.7).

reweighting analysis of single runs yields already reasonable $\sigma^{2}(s, \beta)$ pictures. Therefore, only one more illustration of patching: Figure 10 gives the combined $\sigma^{2}(s, \beta)$ curve from all our four $4 \cdot 16^{3}$ data sets.

Let us first analyse the $L_{t}=4$ data. Table 5 collects FSS fits $(4.4 \mathrm{~b}$ ) (which assume a first order transition) for the $\sigma_{\max }^{2}(s)$ estimates of tables 3 and 4 . Obviously, results from lattices in the range from $L=8$ on are fully compatible with our hypothesis that the transition is first order. If we now treat $\rho$ as a free parameter and perform second order fits (4.4a), we get $\rho=3.08 \pm 0.23$ for the range $L=8-24$ (patched). Although clearly consistent with $\rho=3$, the accuracy is not very restrictive and the error becomes even worse if we restrict the fit to larger lattice sizes. Our best estimate of the latent heat is depicted in figure 11 and yields 


$$
\triangle s=(3.16 \pm 0.06) 10^{-3}, \quad\left(L_{t}=4\right)
$$

Here we have used the $L=8-24$ fit for the patched results, as given in table 5 . The patched results are preferred on the basis of the arguments given in section 2. Monitoring the goodness of fit leads to the choice $L=8-24$. As soon as the goodness of fit is reasonable, there is no statistically significant inconsistency between the smaller and the larger lattices. Still, omitting some smaller lattices in such a situation may decrease the systematic errors. However, this is not relevant anymore within the statistical accuracy. The main effect of omitting the smaller lattices is only an increase of the statistical noise.

Next, we use the $\beta_{\max }(L)$ estimates of tables 3 and 4 as input for the FSS fit (4.7) and arrive at the results of table 6 . Our best estimate of $\beta_{c}$, corresponding to (4.8), is

$$
\beta_{c}^{\text {specific heat }}=5.6933 \pm 0.0004, \quad\left(L_{t}=4\right)
$$

The line of arguments is similar as for our latent heat estimate.

The analysis of the $L_{t}=2$ data is straightforward. The first order nature of the transition is much more pronounced than for $L_{t}=4$. For the $2 \cdot 10^{3}$ lattice the time series and corresponding action histogram are depicted in figures 12 and 13 . This should be compared with figures 1 and 2 . In both cases we have the scale factor $L / L_{t}=5$, but only for $L_{t}=2$ is the two-peak structure immediately clear. For the latent heat as well as for $\beta_{c}$ the $L_{t}=2 \mathrm{FSS}$ fits are now summarized in table 7. For $L=12$ (the only size for which we have two data sets), the results of straightforward error propagation and patching are identical (see tables 3 and 4 ). Thus we only need one table to show these fits. Our best estimates (from $L=6-12$ ) are

$$
\triangle s=(3.09 \pm 0.05) 10^{-2}, \quad\left(L_{t}=2\right)
$$

and

$$
\beta_{c}^{\text {specific heat }}=5.0929 \pm 0.0007, \quad\left(L_{t}=2\right)
$$




\section{PARTITION FUNCTION ZEROS}

Ref. [27] discusses the physical relevance of partition function zeros. Their numerical calculation was pioneered in Refs. [5,28,29,21,9,17], of which [21,17] are concerned with $\mathrm{SU}(3)$ lattice gauge theory. In spin systems the action takes discrete values and the partition function becomes a polynomial in $\exp (\beta)$. Under such circumstances $[28,9]$ the Newton-Raphson method is convenient to calculate the partition function zeros. When the action takes continuous values a time series analysis is more recommendable and we calculate the partition function zeros in two steps: first we scan graphically [29] for the separate zeros of the real and imaginary part. Figure 14 illustrates this for our $4 \cdot 20^{3}$ lattice at $\beta_{0}=5.692$. $\operatorname{Re}(Z)=0$ is denoted by the crosses and $\operatorname{Im}(Z)=0$ by the circles. A partition function zero is obtained when the lines cross. Second, to compute the precise value for the leading zero, we then iterate with AMOEBA [26] (with starting values in a sufficiently small neighborhood of the zero).

Before we can present our SU(3) results we have to clarify some subtle details. For smaller SU(3) lattices we noted in [30] that our empirical action distributions are well described by Gaussian fits. However, a Gaussian distribution does not give rise to zeros in the complex $\beta$ plane. Nevertheless we have reported zeros in [17]. To resolve this paradoxical situation, we first study Gaussian random numbers and proceed then with the analysis of our $\mathrm{SU}(3)$ action distributions.

\subsection{The Gaussian Distribution}

Assume a lattice gauge theory $\mathrm{MC}$ simulation $\left(V_{p}=\right.$ number of plaquettes $)$ and let

$$
x=s-\hat{s} .
$$

For non-critical behaviour the measured probability density for $x$ will be

$$
P(x)=\sqrt{\frac{A}{\pi}} \exp \left(-A x^{2}\right) \quad \text { with } \quad A=\frac{1}{2 \sigma^{2}}=a V_{p} .
$$

By reweighting with $\beta=\beta_{x}+i \beta_{y}$ we obtain the partition function up to a normalization factor:

$$
z(\beta)=\frac{Z(\beta)}{Z\left(\beta_{0}\right)}=\sqrt{\frac{A}{\pi}} \int_{-\infty}^{+\infty} \exp (B x+i C x) \exp \left(-A x^{2}\right) d x
$$


Table 8: Partition function zeros.

\begin{tabular}{|c|c|c|c|c|c|c|c|}
\hline$L_{t} \cdot L^{3}$ & $\beta_{0}$ & $\beta_{x}^{0}$ & $\beta_{y}^{0}$ & $\operatorname{bias}_{x}$ & $\operatorname{bias}_{y}$ & $\triangle \beta_{x}^{\max }$ & $\beta_{y}^{\max }$ \\
\hline $2 \cdot 6^{3}$ & 5.0940 & $5.0910(18)$ & $0.03960(10)$ & $3 \%$ & $-6 \%$ & 0.03150 & 0.05050 \\
\hline $2 \cdot 8^{3}$ & 5.0900 & $5.0905(11)$ & $0.01759(36)$ & $2 \%$ & $-10 \%$ & 0.01290 & 0.02180 \\
\hline $2 \cdot 10^{3}$ & 5.0900 & $5.0927(11)$ & $0.00865(14)$ & $-8 \%$ & $-6 \%$ & 0.00640 & 0.01040 \\
\hline $2 \cdot 12^{3}$ & 5.0920 & $5.0928(08)$ & $0.00502(08)$ & $-4 \%$ & $-9 \%$ & 0.00080 & 0.00500 \\
\hline $2 \cdot 12^{3}$ & 5.0950 & $5.0929(11)$ & $0.00495(11)$ & $23 \%$ & $-28 \%$ & 0.00350 & 0.00570 \\
\hline $4 \cdot 4^{3}$ & 5.5700 & $5.5500(03)$ & $0.09800(04)$ & $-2 \%$ & $-5 \%$ & 0.05000 & 0.10800 \\
\hline $4 \cdot 4^{3}$ & 5.6100 & $5.5600(05)$ & $0.14900(07)$ & $-17 \%$ & $2 \%$ & $0.0000^{*}$ & $0.1230^{*}$ \\
\hline $4 \cdot 4^{3}$ & 5.6400 & $5.6070(06)$ & $0.18500(06)$ & $-7 \%$ & $7 \%$ & $0.0000^{*}$ & $0.1170^{*}$ \\
\hline $4 \cdot 6^{3}$ & 5.5000 & none & & & & & \\
\hline $4 \cdot 6^{3}$ & 5.6400 & $5.6540(10)$ & $0.05200(25)$ & $104 \%$ & $-140 \%$ & 0.04200 & 0.06500 \\
\hline $4 \cdot 6^{3}$ & 5.6450 & $5.6560(05)$ & $0.07570(64)$ & $-50 \%$ & $-22 \%$ & $0.0000^{*}$ & $0.0660^{*}$ \\
\hline $4 \cdot 6^{3}$ & 5.6600 & $5.6420(07)$ & $0.07840(47)$ & $10 \%$ & $35 \%$ & $0.0000^{*}$ & $0.0670^{*}$ \\
\hline $4 \cdot 6^{3}$ & 5.6900 & $5.6450(08)$ & $0.08010(80)$ & $-12 \%$ & $-11 \%$ & $0.0000^{*}$ & $0.0620^{*}$ \\
\hline $4 \cdot 6^{3}$ & 5.7400 & none & & & & & \\
\hline $4 \cdot 8^{3}$ & 5.6000 & none & & & & & \\
\hline $4 \cdot 8^{3}$ & 5.6700 & $5.6747(23)$ & $0.04660(27)$ & $-2 \%$ & $-12 \%$ & $0.0000^{*}$ & $0.0410^{*}$ \\
\hline $4 \cdot 8^{3}$ & 5.6930 & $5.6791(33)$ & $0.04980(42)$ & $5 \%$ & $-16 \%$ & $0.0000^{*}$ & $0.0420^{*}$ \\
\hline $4 \cdot 8^{3}$ & 5.7200 & none & & & & & \\
\hline $4 \cdot 10^{3}$ & 5.6000 & none & & & & & \\
\hline $4 \cdot 10^{3}$ & 5.6800 & $5.6889(14)$ & $0.03010(18)$ & $8 \%$ & $-11 \%$ & $0.0000^{*}$ & $0.0270^{*}$ \\
\hline $4 \cdot 10^{3}$ & 5.6930 & $5.6864(55)$ & $0.02800(81)$ & $7 \%$ & $-7 \%$ & $0.0030^{*}$ & $0.0270^{*}$ \\
\hline $4 \cdot 10^{3}$ & 5.7100 & none & & & & & \\
\hline $4 \cdot 12^{3}$ & 5.6200 & none & & & & & \\
\hline $4 \cdot 12^{3}$ & 5.6810 & none & & & & & \\
\hline $4 \cdot 12^{3}$ & 5.6910 & $5.6896(17)$ & $0.02030(07)$ & $6 \%$ & $-3 \%$ & $0.0000^{*}$ & $0.0180^{*}$ \\
\hline $4 \cdot 12^{3}$ & 5.7030 & none & & & & & \\
\hline $4 \cdot 14^{3}$ & 5.6820 & $5.6886(18)$ & $0.01430(09)$ & $-2 \%$ & $-13 \%$ & $0.0050^{*}$ & $0.0140^{*}$ \\
\hline $4 \cdot 14^{3}$ & 5.6910 & $5.6922(13)$ & $0.01380(07)$ & $0 \%$ & $-13 \%$ & $0.0000^{*}$ & $0.0120^{*}$ \\
\hline $4 \cdot 14^{3}$ & 5.6980 & $5.6859(23)$ & $0.02020(28)$ & $-69 \%$ & $87 \%$ & $0.0000^{*}$ & $0.0130^{*}$ \\
\hline $4 \cdot 16^{3}$ & 5.6830 & $5.6904(16)$ & $0.01010(10)$ & $-11 \%$ & $-15 \%$ & 0.00810 & 0.01060 \\
\hline $4 \cdot 16^{3}$ & 5.6910 & $5.6918(10)$ & $0.01010(06)$ & $-2 \%$ & $-13 \%$ & $0.0000^{*}$ & $0.0094^{*}$ \\
\hline $4 \cdot 16^{3}$ & 5.6920 & $5.6917(10)$ & $0.00960(05)$ & $-2 \%$ & $-14 \%$ & $0.0000^{*}$ & $0.0092^{*}$ \\
\hline $4 \cdot 16^{3}$ & 5.6970 & none & & & & & \\
\hline $4 \cdot 20^{3}$ & 5.6900 & $5.6917(06)$ & $0.00554(22)$ & $-5 \%$ & $-16 \%$ & 0.00230 & 0.00570 \\
\hline $4 \cdot 20^{3}$ & 5.6910 & $5.6915(06)$ & $0.00527(17)$ & $1 \%$ & $-6 \%$ & 0.00120 & 0.00540 \\
\hline $4 \cdot 20^{3}$ & 5.6920 & $5.6929(07)$ & 0.00531 (28) & $-1 \%$ & $-15 \%$ & $0.0000^{*}$ & $0.0051^{*}$ \\
\hline $4 \cdot 24^{3}$ & 5.6910 & $5.6931(07)$ & $0.00270(02)$ & $-29 \%$ & $-35 \%$ & 0.00390 & 0.00420 \\
\hline $4 \cdot 24^{3}$ & 5.6930 & $5.6913(09)$ & $0.00320(04)$ & $32 \%$ & $-49 \%$ & 0.00280 & 0.00390 \\
\hline
\end{tabular}


with (defining $b_{x}$ and $b_{y}$ )

$$
B=V_{p}\left(\beta_{x}-\beta_{0}\right)=: V_{p} b_{x} \quad \text { and } \quad C=V_{p} \beta_{y}=: V_{p} b_{y}
$$

Integration over $x$ gives

$$
z(\beta)=\exp \left(\frac{B^{2}-C^{2}}{4 A}\right) \exp \left(i \frac{B C}{2 A}\right)=|z(\beta)|\left[\cos \left(\frac{B C}{2 A}\right)+i \sin \left(\frac{B C}{2 A}\right)\right] .
$$

We have zeros of the imaginary part for

$$
B=0 \quad \text { and } \quad C=\frac{2 n \pi A}{B},(n=1,2, \ldots)
$$

and of the real part for

$$
C=\frac{(2 n+1) \pi A}{B},(n=0,1,2, \ldots)
$$

Rewriting equations (5.5-5.6) in terms of $b_{x}, b_{y}$ we obtain zeros of $\operatorname{Im}(Z)$ for

$$
b_{x}=0\left(b_{y} \text { arbitrary }\right) \quad \text { and } \quad b_{y}=\frac{2 n \pi A}{\left(V_{p}\right)^{2} b_{x}}=\frac{2 n \pi a}{V_{p} b_{x}},(n=1,2, \ldots)
$$

and of $\operatorname{Re}(Z)$ for

$$
b_{y}=\frac{(2 n+1) \pi A}{\left(V_{p}\right)^{2} b_{x}}=\frac{(2 n+1) \pi a}{V_{p} b_{x}},(n=0,1,2, \ldots) .
$$

The variance $\sigma^{2}(|z(\beta)|)$ is easily calculated to be

$$
\sigma^{2}(|z(\beta)|)=\exp \left(\frac{B^{2}}{A}\right)-|z(\beta)|^{2} .
$$

Assume that a numerical calculation has generated $N$ independent data with the probability density (5.2). We trust with an about $84 \%$ (one sided!) confidence level that the calculation of $z(\beta)$ will not produce artificial zeros in the $(B, C)$-range determined by

$$
\sigma(|\bar{z}(\beta)|)=\frac{\sigma(|z(\beta)|)}{\sqrt{N}} \leq|z(\beta)|
$$

Defining

$$
X=\exp \left(\frac{B^{2}}{4 A}\right) \text { and } Y=\exp \left(\frac{-C^{2}}{4 A}\right)
$$


the equality of (5.8) becomes

$$
X=(N+1)^{1 / 2} Y, \quad \text { where }(N+1)^{-1 / 2} \leq Y \leq 1 .
$$

The argument is only approximate, since numerical results within this confidence radius may have some degree of independence, which is difficult to assess. Here they are just treated as one event.

To give a numerical example, we take $A=1 /\left(2 \sigma^{2}\right)=80,000$ and $V_{p}=6 \cdot 4 \cdot 14^{3}=$ 65,856. We use a Gaussian pseudo random number generator and generate MC data according to the probability density (5.2). Subsequently, a reweighting analysis is done to determine the zeros. For 1,200 and 76,800 independent data, respectively, figures 15 and 16 compare the exact values for the leading zeros of $\operatorname{Im}(Z)$ and $\operatorname{Re}(Z)$ with those from the simulation. Using equation (5.9) the expected range of validity for the numerical results is also indicated and found to be respected by the data. Namely, the apparent crossings (zeros of the partition function) are seen to fall outside the confidence radius. In the Gaussian case we know for sure that this means they are numerical fakes. For our $S U(3)$ data, we shall therefore have to reject any zeros which fall outside the confidence radius.

\section{Table 9: Patching of Partition Function Zeros.}

\begin{tabular}{l|c|c|c|c|l}
\hline$L_{t} \cdot L^{3}$ & $\beta_{x}^{0}$ & $\beta_{y}^{0}$ & {$\left[\beta_{x}^{\min }, \beta_{x}^{\max }\right]$} & $\beta_{y}^{\max }$ & $\#:$ weights. \\
\hline $2 \cdot 12^{3}$ & 5.0928 & $0.00501(7)$ & {$[5.090,5.095]$} & 0.0060 & $2: 0.63,0.37$. \\
& & & & \\
$4 \cdot 4^{3}$ & $5.5520(3)$ & $0.12300(6)$ & none & $0.117^{*}$ & $3: 0.74,0.24,0.02$. \\
$4 \cdot 6^{3}$ & $5.6500(7)$ & $0.07800(5)$ & none & $0.073^{*}$ & $4: 0.29,0.37,0.32,0.02$. \\
$4 \cdot 8^{3}$ & $5.6740(2)$ & $0.04500(2)$ & none & $0.042^{*}$ & $2: 0.68,0.32$. \\
$4 \cdot 10^{3}$ & unstable & unstable & none & none & $2: 0.52,0.48$. \\
$4 \cdot 14^{3}$ & $5.6890(2)$ & $0.01450(6)$ & {$[5.687,5.691]$} & 0.0156 & $3: 0.26,0.44,0.30$. \\
$4 \cdot 16^{3}$ & $5.6915(7)$ & $0.00990(4)$ & {$[5.688,5.693]$} & 0.0106 & $3: 0.09,0.45,0.46$. \\
$4 \cdot 20^{3}$ & $5.6921(5)$ & $0.00550(2)$ & {$[5.690,5.694]$} & 0.0058 & $3: 0.27,0.42,0.31$. \\
$4 \cdot 24^{3}$ & $5.6928(6)$ & $0.00290(2)$ & {$[5.689,5.695]$} & 0.0044 & $2: 0.45,0.55$.
\end{tabular}

We patch those data sets for which results are also reported in table 8 . The weights are in order of increasing $\beta_{0}$. Instead of $\triangle \beta_{x}^{\max }$ we report now $\left[\beta_{x}^{\min }, \beta_{x}^{\max }\right]$ as $\beta_{0}$ is no longer unique. 


\section{2. $S U(3)$ results}

For single runs our leading partition function zeros are collected in table 8 . To estimate whether they are inside or outside the confidence radii defined by equation (5.9), we use the estimated number of independent measurements from table 1 (for instance 44 for the $4 \cdot 24^{3}$ lattices) and $\sigma_{0}^{2}(s)$ from table 2 as width of a fictitious Gaussian distribution. This

leads to the $\triangle \beta_{x}^{\max }$ and $\beta_{y}^{\max }$ values reported in table 8. An example of a zero and its confidence radius is given in figure 17 (also showing the definition of $\triangle \beta_{x}^{\max }$ and $\beta_{y}^{\max }$ ). An asterix in table 8 indicates that the estimated zero lies actually outside the radius of confidence. We see that for the $4 \cdot L^{3}$ lattices most results have problems. The issue is actually quite subtle as repetitions with similar statistics lead to reproducible results. The reason is that for $\beta_{x}=\beta_{0}$ and a Gaussian distribution, $Z\left(\beta_{y}\right)$ falls off exponentially with increasing $\beta_{y}$. As soon as the statistical noise becomes large enough this leads with certainty to a fake crossover of real and imaginary zeros, as illustrated in figures 14 and 16 . Upon a closer inspection of table 8 one may argue that the $2 \cdot 12^{3}, \beta_{0}=5.092$ data set has also a problem. However, for the $L_{t}=2$ distributions we have a pronounced double peak structure and the use of $\sigma_{0}(s)$ from table 2 is not justified. Each of the single Gaussians has a much smaller width, leading to confidence radii larger than those reported in table 8.

To rescue some of our estimates for the $4 \cdot L^{3}$ lattices, we appeal to our patching method. The confidence radii for the patched results are estimated by iterating the equation

$$
\sqrt{\sum_{i=1}^{P}\left(w_{i}\right)^{2} \sigma_{i}^{2}(|\bar{z}(\beta)|)}=\sum_{i=1}^{P} w_{i}\left|z_{i}(\beta)\right| .
$$

The final results are collected in table 9 . Whereas for lattices of size $4 \cdot 4^{3}$ to $4 \cdot 12^{3}$ we still have no conclusive results, we can now determine the leading zero on lattices $4 \cdot L^{3}$ with $L \geq 14$. For these lattices the FSS fit

$$
u_{y}^{0}(L) \sim L^{-1 / \nu} \quad \text { with } \quad u=e^{-\beta}
$$

gives

$$
\nu=0.35 \pm 0.02,\left(L_{t}=4\right)
$$


with a goodness of fit $Q=0.26$. This is consistent with $\nu=1 / d$, i.e. with a first order transition. Fitting $\beta_{x}^{0}(L)$ with the FFS formula (4.7) yields another estimate of the infinite volume critical point:

$$
\beta_{c}^{\text {zeros }}=5.6934 \pm 0.0007,\left(L_{t}=4\right) \text {. }
$$

The fitted range is $L=14-24$ and the goodness of fit is $Q=0.73$.

Compared with the $L_{t}=4$ situation, the $L_{t}=2$ case is unproblematic. All our zeros from $L=6$ to $L=12$ are within the radii of convergence and allow the FSS fit (5.11)

$$
\nu=0.332 \pm 0.004,\left(L_{t}=2\right)
$$

with the acceptable goodness of fit $Q=0.12$. Fitting only the range $L=8-12$ gives $\nu=0.323(6)$ with the goodness of fit $Q=0.30$. The result (5.14) confirms (with less effort) the pioneering work of Karliner et al. [21], who reported $\nu=0.331(6)$ from their more complicated constrained MC calculation of partition function zeros. Our $L=6-12$ estimate of the critical $\beta$ is

$$
\beta_{c}{ }^{\text {zeros }}=5.0930 \pm 0.0007,\left(L_{t}=2\right) \text {. }
$$

with a goodness of fit $Q=0.42$. We see that the $\beta_{c}{ }^{\text {zeros }}$ estimates are well consistent with the $\beta_{c}$ specific heat results of section 4 .

Table 11: Polyakov Susceptibility by standard error propoagation.

\begin{tabular}{l|c|c|c}
\hline$L_{t} \cdot L^{3}$ & \multicolumn{2}{|c|}{$L^{-3} \chi_{\max }(P)$, goodness } & \multicolumn{2}{|c|}{$\beta_{\max }$, goodness } & $\#$ \\
\hline $2 \cdot 12^{3}$ & $1.95(05) 10^{-1}, 0.83$ & $5.0926(06), 0.67$ & 2 \\
& & & \\
$4 \cdot 6^{3}$ & $2.92(05) 10^{-2}, 0.49$ & $5.6957(26), 0.97$ & 4 \\
$4 \cdot 8^{3}$ & $2.37(06) 10^{-2}, 0.03$ & $5.6930(18), 0.86$ & 3 \\
$4 \cdot 10^{3}$ & $2.01(06) 10^{-2}, 0.86$ & $5.6891(12), 0.19$ & 3 \\
$4 \cdot 12^{3}$ & $1.96(09) 10^{-2}, 0.74$ & $5.6913(12), 0.35$ & 2 \\
$4 \cdot 14^{3}$ & $1.75(09) 10^{-2}, 0.17$ & $5.6906(10), 0.07$ & 2 \\
$4 \cdot 16^{3}$ & $1.77(07) 10^{-2}, 0.99$ & $5.6913(06), 0.74$ & 3 \\
$4 \cdot 20^{3}$ & $1.72(06) 10^{-2}, 0.65$ & $5.6917(04), 0.20$ & 3 \\
$4 \cdot 24^{3}$ & $1.67(31) 10^{-2}, 0.34$ & $5.6920(06), 0.05$ & 2
\end{tabular}

Results are calculated from table 10 according to standard error progagation. The last column shows how many data sets were combined. 
Table 10: Results from the Polyakov susceptibility.

\begin{tabular}{|c|c|c|c|c|c|c|}
\hline$L_{t} \cdot L^{3}$ & $\beta_{0}$ & $\bar{P}$ & $L^{-3} \chi_{0}(P)$ & $L^{-3} \chi_{\max }(P)$ & bias & $\beta_{\max }$ \\
\hline $2 \cdot 6^{3}$ & 5.094 & $0.77480(0211)$ & $1.4410^{-1}$ & $1.52(003) 10^{-1}$ & $17 \%$ & $5.0836(25)$ \\
\hline $2 \cdot 8^{3}$ & 5.090 & $0.62510(0378)$ & $1.5810^{-1}$ & $1.60(004) 10^{-1}$ & $38 \%$ & $5.0894(12)$ \\
\hline $2 \cdot 10^{3}$ & 5.090 & $0.38700(0700)$ & $1.4910^{-1}$ & $1.87(006) 10^{-1}$ & $122 \%$ & $5.0936(16)$ \\
\hline $2 \cdot 12^{3}$ & 5.092 & $0.84400(0680)$ & $1.1310^{-1}$ & $1.95(005) 10^{-1}$ & $177 \%$ & $5.0928(07)$ \\
\hline $2 \cdot 12^{3}$ & 5.095 & $0.45420(0872)$ & $1.7910^{-1}$ & $2.01(028) 10^{-1}$ & $53 \%$ & $5.0922(12)$ \\
\hline $4 \cdot 4^{3}$ & 5.570 & $0.27737(0296)$ & $2.6710^{-2}$ & none & & \\
\hline $4 \cdot 4^{3}$ & 5.610 & $0.33716(0404)$ & $3.3410^{-2}$ & none & & \\
\hline $4 \cdot 4^{3}$ & 5.640 & $0.35873(0370)$ & $3.5310^{-2}$ & none & & \\
\hline $4 \cdot 6^{3}$ & 5.500 & $0.09542(0050)$ & $0.2610^{-2}$ & none & & \\
\hline $4 \cdot 6^{3}$ & 5.640 & $0.21732(0437)$ & $1.8110^{-2}$ & none & & \\
\hline $4 \cdot 6^{3}$ & 5.645 & $0.23119(0455)$ & $1.9910^{-2}$ & $3.06(014) 10^{-2}$ & $8 \%$ & $5.6950(06)$ \\
\hline $4 \cdot 6^{3}$ & 5.660 & $0.27107(0430)$ & $2.3810^{-2}$ & $2.90(007) 10^{-2}$ & $5 \%$ & $5.6970(04)$ \\
\hline $4 \cdot 6^{3}$ & 5.690 & $0.37507(0670)$ & $2.8710^{-2}$ & $2.88(007) 10^{-2}$ & $6 \%$ & $5.6960(06)$ \\
\hline $4 \cdot 6^{3}$ & 5.740 & $0.47735(0653)$ & $2.7410^{-2}$ & $3.06(014) 10^{-2}$ & $16 \%$ & $5.6940(05)$ \\
\hline $4 \cdot 8^{3}$ & 5.600 & $0.09220(0114)$ & $2.5910^{-3}$ & none & & \\
\hline $4 \cdot 8^{3}$ & 5.670 & $0.22317(0830)$ & $1.8010^{-2}$ & $2.33(010) 10^{-2}$ & $11 \%$ & $5.6915(35)$ \\
\hline $4 \cdot 8^{3}$ & 5.693 & $0.32431(1003)$ & $2.5010^{-2}$ & $2.50(008) 10^{-2}$ & $12 \%$ & $5.6937(22)$ \\
\hline $4 \cdot 8^{3}$ & 5.720 & $0.45422(0555)$ & $1.6110^{-2}$ & $2.12(012) 10^{-2}$ & $23 \%$ & $5.6922(51)$ \\
\hline $4 \cdot 10^{3}$ & 5.600 & $0.06563(0066)$ & $1.2010^{-3}$ & none & & \\
\hline $4 \cdot 10^{3}$ & 5.680 & $0.21619(0834)$ & $1.7210^{-2}$ & $2.047(093) 10^{-2}$ & $12 \%$ & $5.6901(17)$ \\
\hline $4 \cdot 10^{3}$ & 5.693 & $0.32961(0900)$ & $1.8610^{-2}$ & $1.984(069) 10^{-2}$ & $17 \%$ & $5.6867(18)$ \\
\hline $4 \cdot 10^{3}$ & 5.710 & $0.41699(0815)$ & $1.3310^{-2}$ & $1.997(160) 10^{-2}$ & $30 \%$ & $5.6922(28)$ \\
\hline $4 \cdot 12^{3}$ & 5.620 & $0.05761(0052)$ & $0.1010^{-2}$ & none & & \\
\hline $4 \cdot 12^{3}$ & 5.681 & $0.15435(1002)$ & $1.1610^{-2}$ & $2.04(025) 10^{-2}$ & $28 \%$ & $5.6925(17)$ \\
\hline $4 \cdot 12^{3}$ & 5.691 & $0.27161(1581)$ & $1.9210^{-2}$ & $1.95(009) 10^{-2}$ & $30 \%$ & $5.6903(16)$ \\
\hline $4 \cdot 12^{3}$ & 5.703 & $0.40754(0718)$ & $0.8710^{-2}$ & none & & \\
\hline $4 \cdot 14^{3}$ & 5.682 & 0.15595 (1196) & $1.1410^{-2}$ & $1.64(011) 10^{-2}$ & $30 \%$ & $5.6884(15)$ \\
\hline $4 \cdot 14^{3}$ & 5.691 & $0.24181(1895)$ & $1.8210^{-2}$ & $1.87(012) 10^{-2}$ & $31 \%$ & $5.6920(12)$ \\
\hline $4 \cdot 14^{3}$ & 5.698 & $0.37534(0768)$ & $0.7310^{-2}$ & none & & \\
\hline $4 \cdot 16^{3}$ & 5.683 & 0.10277 (1031) & $0.6810^{-2}$ & $1.78(009) 10^{-2}$ & $102 \%$ & $5.6902(15)$ \\
\hline $4 \cdot 16^{3}$ & 5.691 & $0.22025(1992)$ & $1.7210^{-2}$ & $1.76(012) 10^{-2}$ & $33 \%$ & $5.6915(09)$ \\
\hline $4 \cdot 16^{3}$ & 5.692 & $0.25229(1937)$ & $1.7310^{-2}$ & $1.78(013) 10^{-2}$ & $30 \%$ & $5.6914(09)$ \\
\hline $4 \cdot 16^{3}$ & 5.697 & $0.36245(0793)$ & $0.6810^{-2}$ & none & & \\
\hline $4 \cdot 20^{3}$ & 5.690 & $0.16688(1729)$ & $1.4110^{-2}$ & $1.65(009) 10^{-2}$ & $44 \%$ & $5.6914(05)$ \\
\hline $4 \cdot 20^{3}$ & 5.691 & $0.20443(2119)$ & $1.7110^{-2}$ & $1.76(008) 10^{-2}$ & $55 \%$ & $5.6913(05)$ \\
\hline $4 \cdot 20^{3}$ & 5.692 & $0.19617(2420)$ & $1.6210^{-2}$ & $1.73(012) 10^{-2}$ & $52 \%$ & $5.6926(06)$ \\
\hline $4 \cdot 24^{3}$ & 5.691 & $0.08825(1466)$ & $0.7310 z 2$ & $2.43(085) 10^{-2}$ & $50 \%$ & $5.6933(09)$ \\
\hline $4 \cdot 24^{3}$ & 5.693 & $0.31780(1379)$ & $0.7610^{-2}$ & $1.55(033) 10^{-2}$ & $32 \%$ & $5.6909(08)$ \\
\hline
\end{tabular}

All error bars are calculated with respect to twenty jackknife bins and corrected for the 
Table 12: Patching for the Polyakov Susceptibility.

\begin{tabular}{l|c|c|l}
\hline$L_{t} \cdot L^{3}$ & $L_{\chi \max }^{-3}(P)$ & $\beta_{\max }$ & \multicolumn{1}{|c}{ \# Patches: weights } \\
\hline $2 \cdot 12^{3}$ & $1.95(06) 10^{-1}$ & $5.0927(06)$ & $2: 0.68,0.32$. \\
& & & \\
$4 \cdot 6^{3}$ & $2.95(04) 10^{-2}$ & $5.7007(26)$ & $4: 0.14,0.36,0.33,0.17$ \\
$4 \cdot 8^{3}$ & $2.37(06) 10^{-2}$ & $5.6922(12)$ & $3: 0.37,0.42,0.21$. \\
$4 \cdot 10^{3}$ & $2.00(05) 10^{-2}$ & $5.6885(12)$ & $3: 0.41,0.47,0.12$. \\
$4 \cdot 12^{3}$ & $1.95(08) 10^{-2}$ & $5.6910(13)$ & $2: 0.31,0.69$. \\
$4 \cdot 14^{3}$ & $1.71(06) 10^{-2}$ & $5.6896(07)$ & $3: 0.40,0.41,0.19$. \\
$4 \cdot 16^{3}$ & $1.73(06) 10^{-2}$ & $5.6910(05)$ & $4: 0.15,0.36,0.36,0.13$. \\
$4 \cdot 20^{3}$ & $1.70(05) 10^{-2}$ & $5.6917(04)$ & $3: 0.39,0.34,0.27$. \\
$4 \cdot 24^{3}$ & $1.88(09) 10^{-2}$ & $5.6919(12)$ & $2: 0.46,0.544$.
\end{tabular}

The last column gives the number of patched data sets and the relative weights, ordered by increasing $\beta_{0}$. All error bars are calculated with respect to twenty jackknife bins and corrected for the bias.

Table 13: $L_{t}=4$ FSS fits to $\chi_{\max }(P)$

\begin{tabular}{c|ccc|ccc}
\hline & \multicolumn{2}{|c}{ Standard error } & propagation) & \multicolumn{3}{|c}{ Patching) } \\
$L$ range & $10^{2} a_{1}$ & $a_{2}$ & $Q$ & $10^{2} a_{1}$ & $a_{2}$ & $Q$ \\
\hline $6-14$ & $1.78(05)$ & $2.5(2.0)$ & 0.17 & $1.73(4)$ & $2.7(2.0)$ & 0.04 \\
$6-16$ & $1.76(04)$ & $2.6(2.0)$ & 0.21 & $1.71(4)$ & $2.7(2.0)$ & 0.05 \\
$6-24$ & $1.74(04)$ & $2.6(2.0)$ & 0.33 & $1.71(3)$ & $2.7(2.0)$ & 0.04 \\
$8-16$ & $1.68(06)$ & $3.5(5.0)$ & 0.73 & $1.63(5)$ & $3.8(5.0)$ & 0.46 \\
$8-20$ & $1.68(04)$ & $3.5(4.0)$ & 0.87 & $1.63(4)$ & $3.8(4.0)$ & 0.63 \\
$8-24$ & $1.68(05)$ & $3.5(5.0)$ & 0.94 & $1.66(4)$ & $3.5(4.0)$ & 0.16 \\
$10-24$ & $1.68(05)$ & $3.4(9.0)$ & 0.87 & $1.68(5)$ & $3.2(8.0)$ & 0.11 \\
$12-24$ & $1.65(08)$ & $4.8(2.3)$ & 0.84 & $1.69(7)$ & $2.8(1.9)$ & 0.06 \\
$14-24$ & $1.70(10)$ & $1.8(4.3)$ & 0.91 & $1.79(8)$ & $-2.5(3.0)$ & 0.25
\end{tabular}

Eq. (6.2) is used to fit the $\chi_{\max }(P)$ in tables 11 resp. 12 .

\section{POLYAKOV LINE SUSCEPTIBILITY}

Refs. [15,31] have advocated the FSS analysis of the susceptibility of the projected Polyakov line as a good indicator of the order of the $S U(3)$ phase transition. Since we have measured and recorded the real and imaginary parts of the Polyakov line along with the 
Table 14: $L_{t}=4 \chi(P)$ FSS fits of $\beta_{\max }(L)$

\begin{tabular}{c|ccc|ccc}
\hline & \multicolumn{2}{|c|}{ Standard error propagation) } & \multicolumn{3}{|c}{ (Patching) } \\
$L$ range & $\beta_{c}$ & $a$ & $Q$ & $\beta_{c}$ & $a$ & $Q$ \\
\hline $6-24$ & $5.6914(3)$ & $0.4(5.0)$ & 0.20 & $5.6907(3)$ & $1.1(5.0)$ & 0.005 \\
$8-24$ & $5.6917(4)$ & $-0.7(8.0)$ & 0.31 & $5.6910(4)$ & $-0.2(7.0)$ & 0.060 \\
$10-24$ & $5.6921(4)$ & $-2.8(1.3)$ & 0.94 & $5.6918(4)$ & $-3.4(1.3)$ & 0.600 \\
$12-24$ & $5.6920(5)$ & $-2.3(2.2)$ & 0.87 & $5.6918(6)$ & $-3.9(2.3)$ & 0.440 \\
$14-24$ & $5.6923(6)$ & $-4.4(3.3)$ & 0.98 & $5.6923(7)$ & $-6.7(3.0)$ & 0.740
\end{tabular}

Eq. (4.7) is used to fit the $\beta_{\max }$ in tables 11 resp. 12.

Table 15: $L_{t}=2$ FSS fits of $\chi_{\max }(P)$ and its $\beta_{\max }(L)$

\begin{tabular}{c|ccc|ccc}
\hline$L$ range & $10^{2} a_{1}$ & $a_{2}$ & $Q$ & $\beta_{c}$ & $a$ & $Q$ \\
\hline $6-12$ & $18.8(5.0)$ & $-08.3(01.3)$ & 0.0007 & $5.0942(08)$ & $-2.29(56.0)$ & 0.53 \\
$8-12$ & $21.2(8.0)$ & $-26.0(05.0)$ & 0.7100 & $5.0942(10)$ & $-2.31(97.0)$ & 0.26 \\
$10-12$ & $20.6(1.7)$ & $-19.0(20.0)$ & - & $5.0915(26)$ & $2.10(04.1)$ & -
\end{tabular}

The susceptibility maxima for $L_{t}=2$ are fitted to eq. (6.2) and the corresponding $\beta_{\max }$ to eq. (4.7).

plaquette action, for each of our runs, we can apply the procedures discussed in Section 4 to the spectral-density FSS analysis of this quantity.

We have a time series of measurements of the lattice average of the Polyakov line in the Euclidean time direction, $\Omega=V^{-1} \sum_{x} \Omega_{x}$. These are complex numbers: $\Omega=$ $\operatorname{Re} \Omega+\mathrm{iIm} \Omega=\rho \mathrm{e}^{i \phi}$. (In our definition, $\Omega_{x}$ is larger than in Ref. [15] by the colour factor $3)$.

The projected real part $P$ is computed as

$$
\begin{cases}\text { 1. } & P=\operatorname{Re} \Omega \text { for } \phi \in[-\pi / 3, \pi / 3), \\ \text { 2. } & P=\operatorname{Re} \exp (-i 2 \pi / 3) \Omega \text { for } \phi \in[\pi / 3, \pi), \\ \text { 3. } & P=\operatorname{Re} \exp (i 2 \pi / 3) \Omega \text { for } \phi \in[-\pi,-\pi / 3) .\end{cases}
$$

$P$ provides a clear distinction between the $Z_{3}$-symmetric phase of the theory (where it is close to zero) and any of the three broken phases (where it is nonzero). Since $\phi$ is projected out, there is no cancellation due to tunneling between the three broken phases 
(which makes the time series average $\bar{\Omega}$ vanish on a finite lattice, even above deconfinement). The susceptibility (variance) of $P$ can now be analyzed exactly like the variance of the action was analyzed in Section 4. (To compute the moments of $P$, we calculate the Boltzmann factors from the corresponding action measurements.) The results corresponding to table 2 are collected in table 10. (Remember that our susceptibilities differ from those of Ref. [15] by a factor 9 , because of the normalization of $\Omega_{x}$.)

Table 11 shows the results of the standard error propagation analysis, as applied to the valid results in table 10 . Since the $4 \cdot 4^{3}$ lattices yield no valid results, there is no $4 \cdot 4^{3}$ entry in table 11. Like for the specific heat, the data sets for $L=14,24\left(L_{t}=4\right)$ give poor estimates of $\beta_{\max }$ and the error on $L^{-3} \chi_{\max }$ is large for $L=24$. Again, patching with weights given by eq. (2.9b) (where we put $f_{i}=P_{i}$ ) improves the situation, as can be seen in table 12. However, we notice that the patched $L^{-3} \chi_{\max }$ for $L=24\left(L_{t}=4\right)$ violates the trend in the results for the other $L$.

Regarding the FSS behavior of the susceptibility maxima and of the corresponding values of $\beta$, the same considerations apply as discussed in Section 4. Assuming a first order transition, we fit the data in tables 11 and 12 with the analog of eq. (4.4b):

$$
L^{-3} \chi(P)=a_{1}+a_{2} L^{-3}
$$

Tables 13 and 15 show the results of these fits for $L_{t}=4$ and $L_{t}=2$ respectively. For $L_{t}=4$, we see that the patching data (which are of better quality) are more restrictive than those obtained by error propagation. Our suspicion about the data $L=24$ (presumably insufficient statistics) are confirmed by the $Q$ values for the corresponding fits. In addition, $L=6$ is presumably a too small lattice. The best estimate of the order parameter jump $\triangle P=2 \sqrt{a_{1}}$ should be the one obtained from the patched fit $L=8-24$ ( $Q$ is still acceptable, and while it makes sense to ignore the $L=6$ result we cannot discard our largest lattice $L=24$ even though the statistics is poor.) We get

$$
\triangle P=(0.26 \pm 0.04), \quad\left(L_{t}=4\right)
$$

For $L_{t}=2$ too, the $L=6$ data spoil the first order fit; the best estimate is

$$
\triangle P=(0.92 \pm 0.18), \quad\left(L_{t}=2\right)
$$

from the range $L=8-12$. 
We can also use the $\beta$ values corresponding to the maxima of the susceptibility, in order to obtain $\beta_{c}$ through the fit (4.7). The results of this exercise appear in tables 14 (for $L_{t}=4$ ) and 15 (for $L_{t}=2$ ). Our best estimates for $\beta_{c}{ }^{\text {susc }}$ are

$$
\beta_{c}^{\text {susc }}=5.6918 \pm 0.0004 \quad\left(L_{t}=4\right)
$$

(using $L=10-24$ by patching), and

$$
\beta_{c}^{\text {susc }}=5.0942 \pm 0.0008 \quad\left(L_{t}=2\right)
$$

using $L=6-12$. Note that the $\beta_{c}$ fit selects optimal ranges which are different from the one we used for the fit to the susceptibility maxima. Obviously, this is allowed in principle since the height of the peak and its location in $\beta$ may be independent functions of $L$.

While $\beta_{c}{ }^{\text {susc }}$ for $L_{t}=2$ is seen to be consistent with those estimated from the analysis of the specific heat (cf. eq. (4.11)) and of the partition function zeros (cf. eq. (5.15)), $\beta_{c}$ susc for $L_{t}=4$ is rather small and becomes only consistent on a two $\sigma$ level (cf. eqs. (4.10), (5.13)). This may indicate that with our statistics (presumably due to long time correlations) the Polyakov susceptibility is not accurate enough to allow really precise fits.

\section{SUMMARY AND CONCLUSIONS}

Spectral density methods greatly facilitate accurate FSS calculations. One can calculate pseudocritical couplings precisely and extrapolate towards the infinite volume critical coupling. From the specific heat, the analysis of the partition function zeros and the Polyakov loop susceptibilities we have obtained three estimates of the infinite volume critical $\beta$ which differ somewhat due to remaining systematic errors. In the absence of other strong criteria, one may average these estimates, weighted by their error bars and quote as the final error the best error bar of the single estimates (one can not use error propagation

as all results are obtained from the same configurations). In this way we obtain from (4.9), (5.13) and (6.3)

$$
\beta_{c}=5.6927 \pm 0.0004, \quad\left(L_{t}=4\right)
$$

and from (4.1), (5.15) and (6.4):

$$
\beta_{c}=5.0933 \pm 0.0007, \quad\left(L_{t}=2\right)
$$


The achieved accuracy improves estimates from the pioneering literature [12] by one order of magnitude in the error bar (translating to two orders of magnitude in computer time).

Another notable result are the latent heats (4.8) and (4.11), which are consistent with independent results by other groups [15,32]. Again the spectral density FSS approach works quite well. The possibility to calculate a latent heat and, similarly, an order parameter jump self-consistently is in our opinion the strongest argument in favour of the first order nature of this phase transition. Whereas for $L_{t}=2$ one observes, additionally, a clear double peak structure the $L_{t}=4$ transition is fairly weak and a double peak structure begins (marginally) only to develop from $L=16$ on. It is remarkable that the FSS behavior is nevertheless already quite indicative for the first order nature. This seems to be driven by the increase of the width of the almost Gaussian looking action density distribution.

For $L_{t}=4$ the analysis of the partition function zeros has turned out to be more subtle than previously anticipated. Nevertheless from $L=14$ on the results seem to be conclusive and the obtained estimate (5.12) of the critical exponent $\nu$ is consistent with $\nu=1 / d(d=3)$ and rounds the picture of a weak first order transition. For $L_{t}=2$ the same analysis is fairly unproblematic and the results of [21] are nicely improved and confirmed. The $L_{t}=2$ estimate (5.14) for $\nu$ is, of course, consistent with a first order transition.

\section{ACKNOWLEDGEMENTS}

The MC data were produced on Florida State University's ETA ${ }^{10}$ 's. In addition this work made heavy use of the FSU Physics Department HEP VAX and the Supercomputer Computations Research Institute RISC clusters and their attached storage facilities. This research project was partially funded by the U.S. National Science Foundation under grant INT-8922411 and by the the U.S. Department of Energy under contracts DE-FG0587ER40319 and DE-FC05-85ER2500. Nelson Alves is supported by the German Humboldt foundation.

\section{REFERENCES}

1) Z.W. Salsburg, J.D. Jackson, W. Fickett and W.W. Wood, J. Chem. Phys., 30, (1959), 65.

2) I.R. McDonald and K. Singer, Discuss. Faraday Soc., 43, (1967), 40.

3) J.P. Valleau and D.N. Card, J. Chem. Phys., 57, (1972), 5457. 
4) C.H. Bennett, J. Comp. Phys., 22, (1976), 245.

5) M. Falcioni, E. Marinari, M.L. Paciello, G. Parisi and B. Taglienti, Phys. Lett., 108B, (1982), 331; E. Marinari, Nucl. Phys., B235, [FS11], (1984), 123.

6) G. Bhanot, S. Black, P. Carter and R. Salvador, Phys. Lett., B183, (1987), 331.

7) A.M. Ferrenberg and R.H. Swendsen, Phys. Rev. Lett., 61, (1988), 2635, [erratum 63, (1989), 1658].

8) A.M. Ferrenberg and R.H. Swendsen, Phys. Rev. Lett., 63, (1989), 1196.

9) N.A. Alves, B.A. Berg and R. Villanova, Phys. Rev., B41, (1990), 383.

10) N.A. Alves, B.A. Berg and R. Villanova, Phys. Rev., B43, (1991), 5846.

11) S. Aoki et al. (The Teraflop Collaboration): The QCD Teraflop Project, to be published in Int. J. Mod. Phys. C.

12) J. Kogut et al., Phys. Rev. Lett., 51, (1983); T. Celik, J. Engels and H. Satz, Phys. Lett., 129B, (1983), 323; A. Kennedy et al., Phys. Rev. Lett., 54, (1985), 87; N. Christ and A. Terrano, Phys. Rev. Lett., 56, (1986), 111.

13) P. Bacilieri et. al., Phys. Rev. Lett., 61, (1988), 1545; Phys. Lett., 224B, (1989), 333.

14) A. Ukawa, Proceedings of the LAT89 Capri conference, Nucl. Phys. B, (Proc. Suppl.) 17, (1990), 118.

15) M. Fukugita, M. Okawa and A. Ukawa, Nucl. Phys., B337, (1990), 181.

16) B.A. Berg, R. Villanova and C. Vohwinkel, Phys. Rev. Lett., 62, (1989), 2433.

17) N.A. Alves, B.A. Berg and S. Sanielevici, Phys. Rev. Lett., 64, (1990), 3107.

18) A.D. Sokal, " Monte Carlo Methods in Statistical Mechanics: Foundations and New Algorithms", preprint, New York University (1989).

19) B.A. Berg and J. Stehr, Z. Phys, C9, (1981), 333.

20) H. Flyvbjerg and H.G. Peterson, J. Chem. Phys., 91, (1989), 461.

21) M. Karliner, S. Sharpe and Y. Chang, Nucl. Phys., B302, (1988), 204.

22) B.A. Berg, A. Devoto and C. Vohwinkel, Comp. Phys. Commun., 51, (1988), 331.

23) B.A. Berg, preprint, SCRI 90-100, to be published in Comp. Phys. Commun. 
24) M.E. Fisher, in Nobel Symposium 24, B. Lundquist and S. Lundquist (editors), Academic Press, New York, 1974.

25) B.L. Van der Waerden, Mathematical Statistics (Springer, New York, 1969).

26) W. Press et al., Numerical Recipes, Cambridge University Press, London, 1986.

27) C. Itzykson, R.B. Pearson and J.B. Zuber, Nucl. Phys., B220, [FS8], (1983), 415.

28) G. Bhanot, S. Black, P. Carter and R. Salvador, Phys. Lett., B183, (1987), 331.

29) K. Bitar, Nucl. Phys., B300, [FS22], (1988), 61.

30) N.A. Alves, B.A. Berg and S. Sanielevici, Phys. Lett., 241B, (1990), 557.

31) M. Fukugita, H. Mino, M. Okawa and A. Ukawa, Nucl. Phys. B, (Proc. Suppl.), 20, (1991), 258.

32) F. Brown, N. Christ, Y. Deng, M. Gao and T. Woch, Phys. Rev. Lett., 61, (1988), 2058. 